\section{ICU: Lactoferrin gegen nosokomiale Infektionen?}

Muscedere J et al. Prevention of Nosocomial Infections in Critically III Patients With Lactoferrin: A Randomized, Double-Blind, PlaceboControlled Study. Crit Care Med 2018; doi:10.1097/CCM.0000000000003294

Nosokomiale Infektionen stellen ein hohes Risiko für beatmete Intensivpatienten dar und können das Behandlungsergebnis negativ beeinflussen. Da das körpereigene Glykoprotein Lactoferrin mit dem angeborenen Immunsystem in Verbindung gebracht wird und über die Bindung von Eisen das Wachstum von Bakterien hemmen kann, scheint hier präventives Potenzial zu liegen. Muscedere und Team wollten dem nachgehen, und haben eine Phase-II-Studie durchgeführt.

Die invasive Beatmung von intensivpflichtigen Patienten stellt für Bakterien eine ausgezeichnete Eintrittspforte dar, die Entwicklung nosokomialer Infektionen kann das Outcome erheblich negativ beeinflussen. Da erste klinische Untersuchungen Hinweise dafür liefern, dass das körpereigene Glykoprotein Lactoferrin solche Infektionen möglicherweise verhindern könnte, haben Muscedere und seine Kollegen nun eine entsprechende klinische, randomisierte, doppelblinde und placebokontrollierte Phase-IIStudie durchgeführt.

Lactoferrin kommt als wichtiger Akteur in der angeborenen Immunabwehr vor und kann über die Bindung von Eisen das Wachstum von Bakterien hemmen. Die Forscher stellten vorab die Hypothese auf, dass eine orale oder nasogastrale Gabe von Lactoferrin bei Intensivpatienten unter invasiver Beatmung die Häufigkeit von nosokomialen Infektionen reduzieren, das Behandlungsergebnis verbessern und schließlich den Antibiotikabedarf verringern würde.

Die Studie fand zwischen November 2013 und Dezember 2017 an 5 kanadischen Krankenhäusern der Regelversorgung statt. Einschlusskriterien waren neben einer intensivpflichtigen Behandlung eine invasive Beatmung für mindestens 48 Stunden zum Zeitpunkt der Randomisierung sowie ein Patientenalter ab 18 Jahren. Die Studienautoren definierten weiterhin folgende Ausschlusskriterien:

- Intensivbehandlung für weniger als 72 Stunden,

- Kontraindikation zur oralen oder nasogastralen Aufnahme,

- kein Zugang zur Mundhöhle,

- Allergie oder Empfindlichkeit gegenüber Lactoferrin oder Rinderprotein oder Milch, Immunsuppression,

- Lebenserwartung unter 6 Monaten, Schwangerschaft oder Stillzeit.

- Geeignete Patienten wurden anschließend einer von 2 Studiengruppen zugeteilt:

- Interventionsgruppe (IG): orale und nasogastrale Gabe von $2 \mathrm{~g}$ Rinderlactoferrin in $200 \mathrm{ml}$ Wasser in 4 Dosen pro Tag bis zum 28. Tag nach Randomisierung.

- Placebogruppe (PG): Gabe von $200 \mathrm{ml}$ Wasser in 4 Dosen pro Tag bis zum 28. Tag nach Randomisierung.

Neben der Intervention erhielten alle Patienten die Standardbehandlung.

Als primären klinischen Endpunkt definierten die Studienautoren antibiotikafreie Tage bis zum 28. Tag nach Randomisierung. Die sekundären Endpunkte waren unter anderem

- Machbarkeit,

- Antibiotikabedarf,

- Veränderungen der Organfunktion gemessen mit dem Sequential Organ Failure Assessment (SOFA) Score,

- Krankenhausverweildauer,

- Verweildauer auf Intensivstation,

- 90-Tage-Sterblichkeit

- sowie die Konzentrationen sämtlicher Zytokine.

\section{Keinerlei Gruppenunter- schiede}

638 Patienten erfüllten die Kriterien, 214 wurden randomisiert und 212 von ihnen nahmen schließlich an der Studie teil. Sie waren durchschnittlich 66,3 (IG) bzw. 62,5 (PG) Jahre alt, 44,9 bzw. 48,6\% von ihnen weiblich. Patienten der Interventionsgruppe waren älter, hatten in gängi- gen Scores eine größere Krankheitsschwere und litten eher an einer schweren Sepsis als Mitpatienten der Placebogruppe.

Den primären klinischen Endpunkt konnten die Forscher auf 18,5 Tage für die Interventionsgruppe und auf 17,3 Tage für die Placebogruppe beziffern. Dieser Unterschied war dabei statistisch und klinisch nicht signifikant. Insgesamt stellten die Forscher bei 27,8\% der Patienten die Entwicklung einer nosokomialen Infektion im Studienzeitraum fest. Auch hier gab es keine Unterschiede zwischen den Studiengruppen.

Da die Studienautoren auch für alle anderen klinischen Endpunkte einschließlich der Konzentrationen der zahlreich bestimmten Zytokine keine bedeutsamen Differenzen feststellen konnten, halten sie in ihrem Diskussionsteil das Glykoprotein Lactoferrin für nicht überzeugend wirksam zur Prävention nosokomialer Infektionen und sehen von weiteren klinischen Studien ab.

FAZIT

In dieser Phase-II-Studie hatte die präventive orale und gastrale Gabe des Glykoproteins Lactoferrin keinen Einfluss auf die Häufigkeit nosokomialer Infektionen bei invasiv beatmeten Intensivpatienten. Da alle Endpunkte - inklusive Antibiotikabedarf, Krankenhausverweildauer und die Konzentrationen sämtlicher Zytokine - zwischen beiden Studiengruppen vergleichbar waren, sehen die Autorinnen/Autoren von weiteren klinischen Studien zur Wirksamkeit von Lactoferrin ab.

Dipl.-Psych. Annika Simon, Hannover 